\title{
Options and Efficiency in Multiperiod Security Markets*
}

\author{
Alexandre M. Baptista \\ University of Minnesota
}

November 15, 1999

*I am grateful to Jan Werner for his guidance and very helpful comments. This paper also benefited from the comments of Luca Benzoni and the seminar participants at the 1999 Midwest Math. Econ. Meetings. I am responsible for all remaining errors. Financial support from Sub-Programa Ciência e Tecnologia do Segundo Quadro Comunitário de Apoio is gratefully acknowledged. Please address correspondence to Alexandre Baptista, University of Minnesota, Carlson School of Management, Department of Finance, 321-19th Avenue South, Minneapolis, MN 55455. Phone: 612-626-9698. Fax: 612-626-1335. E-mail: abaptista@csom.umn.edu. 


\title{
Options and Efficiency in Multiperiod Security Markets
}

\begin{abstract}
We extend the result of Ross (1976, Quart. J. Econ., 90, 75-89) that European options generate complete markets from the single-period to a multiperiod setting. We find that multiperiod European options on a trading strategy generate dynamic completeness for every arbitrage-free price process, provided that the trading strategy has non-negative terminal dividends and separates states at the terminal date. Furthermore, we show that if the uncertainty and information structure in an economy are such that the number of immediate successors of every non-terminal event is non-decreasing over time, then multiperiod European options on a trading strategy generate dynamic completeness for almost every arbitrage-free price process under a significantly weaker condition on the trading strategy's terminal dividends. This condition requires the trading strategy to have non-negative terminal dividends and to separate states at the terminal date conditional on the information available at the previous date. Finally, we examine the minimum number of options generating dynamic completeness for almost every arbitrage-free price process.

Journal of Economic Literature Classification Numbers: D52, D61.

Keywords: Options; Incomplete Markets; Dynamic Spanning
\end{abstract}




\section{Introduction}

European options have been recognized as appropriate derivative securities to generate complete markets, i.e., full spanning of the contingencies in an economy. ${ }^{1}$ Complete markets lead to an efficient allocation of risk in equilibrium. Ross [28] showed that in a single-period setting, European options on a portfolio having non-negative dividends and separating states (i.e., paying different dividends in different states) generate complete markets. In this paper we extend Ross' result to multiperiod security markets.

There is a simple way of extending Ross' result. An appropriate set of single-period European options available at every trading date generates dynamic completeness (see Friesen [14]). However, in practice options are retraded (i.e., multiperiod) and few maturity dates are available for trade. It would be costly to issue options at every trading date. ${ }^{2}$

In contrast, we consider multiperiod European options issued at the initial date with maturity at the terminal date. We investigate dynamic completeness with multiperiod European options for every arbitrage-free price process (for the primitive securities and the options); Fxamining a particular arbitrage-free price process would not be satisfactory since one may obtain dynamic completeness for some security prices but not for others. ${ }^{3}$

We introduce two notions of multiperiod separation. A trading strategy separates states at the terminal date if its terminal dividends provide per se a full description of the state of nature. A substantially weaker notion of separation is that of a trading strategy that conditionally separates states at the terminal date, i.e., a trading strategy whose terminal dividends provide a full description of the state of nature given the information available at

1 See Arditti and John [1], Banz and Miller [4], Breeden [5], Breeden and Litzenberger [6], Brown and Ross [7], Demange and Laroque [10], Green and Jarrow [15], Hakansson [16, 17, 18], John [19, 20], and Schrems [29].

2 A difficulty that may arise when options are issued is the existence of equilibrium. See Kahn and Krasa [21] and Polemarchakis and $\mathrm{Ku}[26]$ for robust examples of non-existence of equilibrium with American options in a single-period setting and with European options in a multiperiod setting, respectively.

3 See, for example, Magill and Quinzii [23, pp. 235-237]. Detemple and Selden [11] showed that if a nonredundant option is issued, then the prices of the primitive securities generically change. Therefore, it is also not appropriate to take as given the incomplete markets prices of the primitive securities. 
the previous date.

Our first major result says that multiperiod European options on a trading strategy having non-negative terminal dividends and separating states at the terminal date generate dynamic completeness for every arbitrage-free price process. An interesting asset pricing implication is that multiperiod European options can be used to price any security (in particular, single-period European options). While the assumption of separation at the terminal date can be weakened, we show that in general, multiperiod European options on a trading strategy having non-negative terminal dividends and conditionally separating states at the terminal date do not generate dynamic completeness for every arbitrage-free price process.

The notion of dynamic completeness for every arbitrage-free price process is restrictive. When there are no multiperiod European options on a trading strategy generating dynamic completeness for every arbitrage-free price process, there may still exist multiperiod European options on that trading strategy generating dynamic completeness for "almost every" arbitrage-free price process. Furthermore, the number of multiperiod European options generating dynamic completeness for every arbitrage-free price process can be relatively large.

For that reason we then examine dynamic completeness for "almost every" arbitrage-free price process, which we refer to as generic dynamic completeness. Our second major result says that when the number of immediate successors of every non-terminal event is nondecreasing over time, multiperiod European options on a trading strategy generate generic dynamic completeness under a significantly weaker condition on the trading strategy's terminal dividends. This condition requires the trading strategy to have non-negative terminal dividends and to conditionally separate states at the terminal date. We show that this result does not extend to event trees in which the number of immediate successors of every non-terminal event decrease over time.

We find that the minimum number of multiperiod European options on a trading strategy 
generating generic dynamic completeness can be relatively large. We then show that either multiperiod European options on several trading strategies or exotic options on a single trading strategy can substantially reduce the minimum number of options generating generic dynamic completeness.

Our conclusions are different from those reached by Bajeux-Besnainou and Rochet [3] in a multinomial framework generalizing the economy of Cox, Ross, and Rubinstein [8]. Bajeux-Besnainou and Rochet concluded in pp. 1-2 that European options "are not a good instrument for dynamic spanning" and that "only path-dependent ('exotic') options may generate dynamic spanning." Our findings about the ability of few options to generate generic dynamic completeness are related with the result of Kreps [22]. Kreps showed that "almost every" set of long-lived securities with cardinality equal to the largest number of immediate successors of an event among all non-terminal events generates dynamic completeness. ${ }^{4}$

This paper is organized as follows. In Section 2 we outline the model. In Section 3 we investigate the existence of multiperiod European options generating dynamic completeness for every arbitrage-free price process. In Section 4 we examine generic dynamic completeness with multiperiod European options. In Section 5 we study the minimum number of multiperiod options generating generic dynamic completeness. Section 6 concludes. All proofs are given in the Appendix.

\section{Dynamically Complete Markets}

Consider an event tree economy as described, for example, in Duffie [12, Chapter 2]. Let $\mathbb{T} \equiv\{0, \ldots, T\}$, where $T \in \mathbb{N}$ is the number of periods in an event tree. The uncertainty and the information structure are described by a filtered probability space $\left(\Omega, \digamma,\left\{\digamma_{t}\right\}_{t \in \mathbb{T}}, P\right)$, where $\Omega$ is a finite set of states of nature, $\digamma=\digamma_{T}=2^{\Omega}$, and $\digamma_{t}$ is the information available at time $t \in \mathbb{T} .^{5}$ Let $\Xi\left(\Xi_{-T}\right)$ be the set of events at all dates $t>0$ (respectively, $t<T$ ). Let

4 See Duffie and Huang [13] for a continuous-time framework.

5 A filtered probability space is a quadruple $\left(\Omega, \digamma,\left\{\digamma_{t}\right\}_{t \in \mathbb{T}}, P\right)$, where $\Omega$ is a state space, $\digamma$ is a sigma algebra of subsets of $\Omega,\left\{\digamma_{t}\right\}_{t \in \mathbb{T}}$ is a non-decreasing sequence of sub-sigma algebras of $\digamma$, and $P$ is a probability 
$k \equiv \sharp(\Xi)$ and $n_{\xi}$ be the number of immediate successors of event $\xi \in \Xi_{-T}$.

There are $J \in \mathbb{N}$ primitive securities. Let $\mathbb{J} \equiv\{1, \ldots, J\}$. The dividend process of security $j \in \mathbb{J}$ is $d^{j}$, which is assumed adapted. ${ }^{6}$ Let $d \equiv\left\{d^{j}\right\}_{j \in \mathbb{J}}$. Let $p \equiv\left\{p^{j}\right\}_{j \in \mathbb{J}}$ denote an ex-dividend price process for the primitive securities.

A trading strategy is denoted by $h \in \mathbb{H}$, where $\mathbb{H}$ is the set of all $\mathbb{R}^{J}$-valued processes. We use the term "portfolio" instead of "trading strategy" when $T=1$. Frictionless short-sales are allowed. The dividend process $d^{h}(p)$ generated by trading strategy $h \in \mathbb{H}$ under price process $p$ is defined by

$$
d_{t}^{h}(p)=h_{t-1} \cdot\left(p_{t}+d_{t}\right)-h_{t} \cdot p_{t}, \forall t \in \mathbb{T}
$$

where $h_{-1} \equiv 0$.

An arbitrage for a dividend-price process $(d, p)$ is a trading strategy $h \in \mathbb{H}$ such that $d^{h}(p) \geq 0$ and $d^{h}(p) \neq 0$. We say that price process $p$ is arbitrage-free if there exists no arbitrage for $(d, p)$. Whenever $p$ is arbitrage-free, we have

$$
p_{T}^{j}=0, \forall j \in \mathbb{J}
$$

It follows from (1) and (2) that the terminal dividend $d_{T}^{h}$ does not depend on $p$ for every trading strategy $h \in \mathbb{H}$.

We denote a consumption process by $c \in \mathbb{C}$, where $\mathbb{C}$ is the set of all $\mathbb{R}$-valued processes. The following notion of dynamic completeness is standard.

Definition 1. A dividend-price process $(d, p)$ is dynamically complete if, for every consumption process $c \in \mathbb{C}$, there is a trading strategy $h \in \mathbb{H}$ such that $d_{t}^{h}(p)=c_{t}, t>0$. Otherwise, $(d, p)$ is incomplete.

We use the term "complete" instead of "dynamically complete" when $T=1$. Let $D_{t+1}\left(\xi_{t}\right)$ $\left[P_{t+1}\left(\xi_{t}\right)\right]$ be a $\left(J \times n_{\xi_{t}}\right)$ matrix of one-period dividends (respectively, prices) for the primitive measure on $(\Omega, \digamma)$.

6 A process $X=\left\{X_{t}\right\}_{t \in \mathbb{T}}$ is adapted if $X_{t}: \Omega \rightarrow \mathbb{R}$ is measurable with respect to $\left(\Omega, \digamma_{t}\right)$ for every $t \in \mathbb{T}$. Throughout, all processes are assumed to be adapted. 
securities at event $\xi_{t} \in \Xi_{-T}$. We say that a dividend-price process $(d, p)$ is one-period complete at event $\xi_{t} \in \Xi_{-T}$ if

$$
\operatorname{rank}\left[D_{t+1}\left(\xi_{t}\right)+P_{t+1}\left(\xi_{t}\right)\right]=n_{\xi_{t}} .
$$

If $(d, p)$ is one-period complete at $\xi_{t}$ for every $\xi_{t} \in \digamma_{t}$, where $t<T$, then $(d, p)$ is one-period complete at time $t$. It follows from $(2)$ and (3) that one-period completeness of $(d, p)$ at time $T-1$ does not depend on $p$.

The following is a well known result.

Lemma 1. A dividend-price process $(d, p)$ is dynamically complete if and only if $(d, p)$ is one-period complete at every event $\xi \in \Xi_{-T}$.

There is an infinite number of distinct arbitrage-free price processes for every dividend process $d$. It follows from $(3)$ that one-period completeness of a dividend-price process $(d, p)$ at an event $\xi_{t} \in \digamma_{t}$, where $t \leq T-2$, depends on $p$. Using Lemma 1, it may be the case that $(d, p)$ is dynamically complete but $\left(d, p^{\prime}\right)$ is incomplete for $p \neq p^{\prime}$. Since security prices determine whether a dividend-price process is dynamically complete, Definition 1 is not satisfactory for the purposes of our paper.

We now introduce a notion of dynamic completeness for every arbitrage-free price process.

Definition 2. A dividend process $d$ is dynamically complete if $(d, p)$ is dynamically complete for every arbitrage-free price process p.

Under standard monotonicity assumptions on consumer preferences, if dividend process $d$ is dynamically complete, then every Radner [27] equilibrium consumption allocation with $d$ is an Arrow-Debreu [2, 9] equilibrium consumption allocation and, therefore, it is Pareto optimal.

We say that a dividend process $d$ is one-period complete at event $\xi \in \Xi_{-T}$ if $(d, p)$ is one-period complete at $\xi$ for every arbitrage-free price process $p$. If $d$ is one-period complete 
at $\xi_{t}$ for every $\xi_{t} \in \digamma_{t}$, where $t<T$, then $d$ is one-period complete at time $t$.

\section{Dynamic Completeness with Options}

Suppose that the dividend process of the primitive securities is not dynamically complete. The question we are interested in is whether amending the primitive securities with multiperiod options generates a dynamically complete dividend process. In a single-period setting, Ross showed that options on portfolios are better suited to generate complete markets than options on individual securities. Therefore, we consider in our multiperiod setting options on trading strategies.

A multiperiod European call (put) option on trading strategy $h \in \mathbb{H}$ with maturity at time $T$ and exercise price $K \in \mathbb{R}_{+}$(i.e., negative exercise prices are precluded in our analysis), is a security with terminal dividends given by $\max \left(0, d_{T}^{h}-K\right)\left[\operatorname{respectively,~} \max \left(0, K-d_{T}^{h}\right)\right]$, and zero dividends at any other trading date. Hence, we allow options on (i) individual securities, (ii) buy-and-hold trading strategies involving more than one security (i.e., trading strategies where the position in every security is fixed along an event tree), and (iii) trading strategies where the position in some security changes as the uncertainty is resolved along: an event tree. We denote a dividend-price process for a set of multiperiod European options on trading strategy $h \in \mathbb{H}$ (primitive security $j \in \mathbb{J})$ by $\left(e^{h}, q\right)$ [respectively, $\left(e^{j}, q\right)$.

In a single-period framework, European options on a portfolio having non-negative dividends and separating states generate complete markets. To study dynamic completeness with multiperiod options we also need a notion of multiperiod separation.

Definition 3. A trading strategy $h \in \mathbb{H}$ separates states at time $T$ if $d_{T}^{h}\left(\xi_{T}\right) \neq d_{T}^{h}\left(\xi_{T}^{\prime}\right)$ for every $\xi_{T}, \xi_{T}^{\prime} \in \digamma_{T}, \xi_{T} \neq \xi_{T}^{\prime}$

In other words, trading strategy $h$ separates states at time $T$ if its terminal dividends provide per se a full description of the state of nature (i.e., if the cardinality of the support 
of $h$ 's terminal dividends coincides with the number of terminal events). The property given in Definition 3 may also be called (unconditional) separation at the terminal date.

Although coinciding with the single-period notion of a portfolio separating states when $T=1$, our definition of a trading strategy separating states at the terminal date is restrictive in multiperiod markets. For example, a stock in the multiperiod binomial economy of Cox, Ross, and Rubinstein does not separate states at the terminal date.

We now provide a substantially weaker notion of multiperiod separation.

Definition 4. A trading strategy $h \in \mathbb{H}$ separates states at time $T$ conditional on $\digamma_{T-1}$ if, for every $\xi_{T-1} \in \digamma_{T-1}, d_{T}^{h}\left(\xi_{T}\right) \neq d_{T}^{h}\left(\xi_{T}^{\prime}\right)$ for every $\xi_{T}, \xi_{T}^{\prime} \subset \xi_{T-1}, \xi_{T} \neq \xi_{T}^{\prime}$.

In other words, trading strategy $h$ separates states at time $T$ conditional on $\digamma_{T-1}$ if, given the information available at time $T-1$, its terminal dividends provide a full description of the state of nature. The property given in Definition 4 may also be called conditional separation at the terminal date.

When $T=1$, our definition of a trading strategy conditionally separating states at the terminal date coincides with the single-period notion of a portfolio separating states. Using Definitions 3 and 4 , if trading strategy $h$ separates states at time $T$, then it separates states at time $T$ conditional on $\digamma_{T-1}$.

We now illustrate our two notions of multiperiod separation with an example.

Example 1. Consider a two-period event tree with $\Omega=\{1,2,3,4\}, \xi_{11}=\{1,2\}, \xi_{12}=\{3,4\}$, and $\xi_{2 s}=\{s\}$ for every $s \in \Omega$. There are three securities. Security $A$ has $d_{2}^{A}\left(\xi_{21}\right)=4$, $d_{2}^{A}\left(\xi_{22}\right)=3$, and $d_{2}^{A}\left(\xi_{2 s}\right)=2$ for every $s \in\{3,4\}$. Security B has $d_{2}^{B}\left(\xi_{21}\right)=4, d_{2}^{B}\left(\xi_{2 s}\right)=3$ for every $s \in\{2,3\}$, and $d_{2}^{B}\left(\xi_{24}\right)=2$. Security $\mathrm{C}$ has $d_{2}^{C}\left(\xi_{21}\right)=4, d_{2}^{C}\left(\xi_{22}\right)=3, d_{2}^{C}\left(\xi_{23}\right)=2$, and $d_{2}^{C}\left(\xi_{24}\right)=1$ (see Figure 1). Security A does not separate states at time 2 conditional on $\digamma_{1}$ and, therefore, does not separate states at time 2. Security B separates states at time 


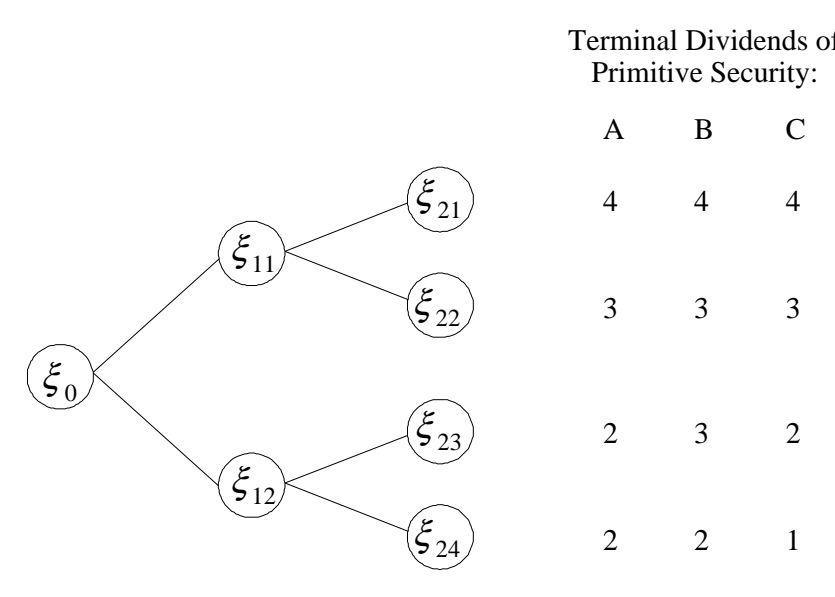

Figure 1: Security A does not conditionally separate states at time 2 and, therefore, does not separate states at time 2 . Security B conditionally separates states at time 2 but does not separate states at time 2. Security $\mathrm{C}$ separates states at time 2 and, therefore, conditionally separates states at time 2 .

2 conditional on $\digamma_{1}$ but does not separate states at time 2. Finally, security $\mathrm{C}$ separates states at time 2 and, therefore, separates states at time 2 conditional on $\digamma_{1}$. $\square$

Let $\mathbb{H}_{+}^{u}$ be the set of trading strategies $h \in \mathbb{H}$ having non-negative terminal dividends and (unconditionally) separating states at the terminal date. Observe that $\mathbb{H}_{+}^{u}$ depends on the dividend process $d$.

The following is our first major result.

Proposition 1. For every trading strategy $h \in \mathbb{H}_{+}^{u}$, there exist multiperiod European options on $h$ such that $\left(d, e^{h}\right)$ is dynamically complete.

It follows from Proposition 1 that under the assumption of separation at the terminal date, there exists a trading strategy on which all multiperiod European options can be written to generate a dynamically complete dividend process.

One might conjecture that multiperiod European options on a trading strategy having non-negative terminal dividends and conditionally separating states at the terminal date generate a dynamically complete dividend process. The following example shows that such conjecture is not correct. 


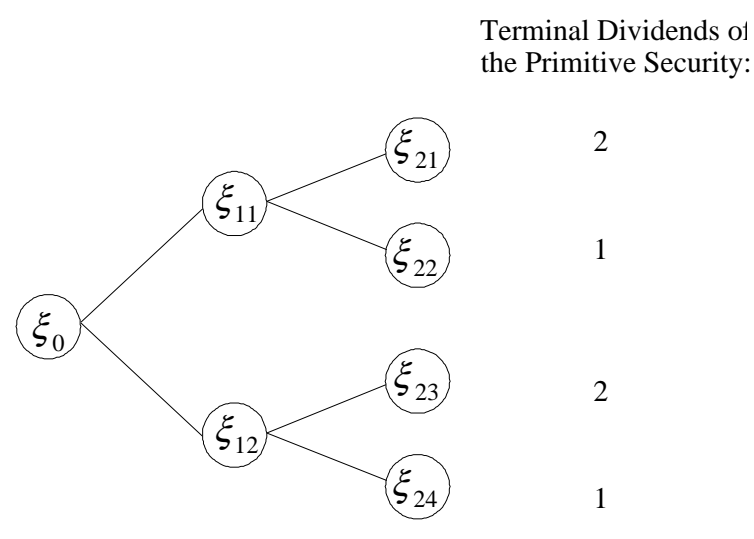

Figure 2: An example where although there are no multiperiod European options on the primitive security generating a dynamically complete dividend process, there exists a multiperiod European option on the primitive security generating a dynamically complete dividend-price process for "almost every" arbitrage-free price process.

Example 2. Consider the event tree of Example 1. Assume that there is a single primitive security with $d_{2}\left(\xi_{2 s}\right)=2$ for every $s \in\{1,2\}, d_{2}\left(\xi_{2 s}\right)=1$ for every $s \in\{3,4\}$, and zero dividends at time 1 (see Figure 2). Note that the primitive security has non-negative terminal dividends, separates states at time 2 conditional on $\digamma_{1}$ but does not separate states at time 2 . Without loss of generality, suppose that a multiperiod European put option on the primitive security with exercise price equal to 2 is issued at time 0. Any further multiperiod European option on the primitive security is redundant. The $(2 \times 4)$ matrix of terminal dividends generated by the primitive security and the option is

$$
\left[\begin{array}{llll}
2 & 1 & 2 & 1 \\
0 & 1 & 0 & 1
\end{array}\right]
$$

It follows from $(3)$ that $\left(d, e^{1}\right)$ is one-period complete at time 1 . Using $(3),\left(d, e^{1}, p, q\right)$ is one-period complete at time 0 if and only if

$$
\operatorname{rank}\left\{\left[\begin{array}{ll}
p_{1}\left(\xi_{11}\right) & p_{1}\left(\xi_{12}\right) \\
q_{1}\left(\xi_{11}\right) & q_{1}\left(\xi_{12}\right)
\end{array}\right]\right\}=2 .
$$

Note that (4) does not hold for every arbitrage-free price process $(p, q)$ with $q_{1}=\alpha \cdot p_{1}$, where $0<\alpha<1$ and $p_{1}>0$. Hence, $\left(d, e^{1}, p, q\right)$ is not one-period complete at time 0 for 
some arbitrage-free price process $(p, q)$. Using Lemma $1,\left(d, e^{1}, p, q\right)$ is incomplete for some arbitrage-free price process $(p, q)$. It follows from Definition 2 that $\left(d, e^{1}\right)$ is not dynamically

complete. However, we shall show in Proposition 2 that $\left(d, e^{1}, p, q\right)$ is dynamically complete for "almost every" arbitrage-free price process $(p, q)$.

Although Example 2 showed that there may not exist multiperiod European options on a trading strategy having non-negative terminal dividends and conditionally separating states at the terminal date that generate a dynamically complete dividend process, we show in Appendix $\mathrm{C}$ that the separation assumption imposed in Proposition 1 can be weakened.

The notion of a dynamically complete dividend process is restrictive. First, as Fxample 2 anticipated, when there are no multiperiod European options on a trading strategy generating a dynamically complete dividend process, there may still exist multiperiod European options on that trading strategy generating a dynamically complete dividend-price process for "almost every" arbitrage-free price process. Second, the number of multiperiod European options generating a dynamically complete dividend process can be relatively large.

\section{Generic Dynamic Completeness with Options}

Suppose that the primitive securities do not generate a dynamically complete dividendprice process for "almost every" arbitrage-free price process (for example, if the number of primitive securities is smaller than the number of immediate successors of an event). The question we are interested in is whether amending the primitive securities with multiperiod options on a trading strategy generates a dynamically complete dividend-price process for "almost every" arbitrage-free price process.

We use an implication of the absence of arbitrage, i.e., the existence of strictly positive event prices, to parameterize security prices. Then, we use this parameterization to introduce a notion of dynamic completeness for "almost every" arbitrage-free price process.

The following is a well-known characterization of the absence of arbitrage. 
Lemma 2. A dividend-price process $(d, p)$ is arbitrage-free if and only if, for every event $\xi_{t} \in \Xi_{-T}$, there exist event prices $\psi\left(\xi_{t}\right) \in \mathbb{R}_{++}^{n_{\xi_{t}}}$ such that

$$
p_{t}\left(\xi_{t}\right)=\sum_{\xi_{t+1} \subset \xi_{t}} \psi_{\xi_{t+1}} \cdot\left[d_{t+1}\left(\xi_{t+1}\right)+p_{t+1}\left(\xi_{t+1}\right)\right]
$$

where $\psi_{\xi_{t+1}}$ is the price of an event $\xi_{t+1} \subset \xi_{t}$ at $\xi_{t} \in \digamma_{t}$.

Using Lemma 2, we can parameterize arbitrage-free price processes by strictly positive event prices. The arbitrage-free price process associated with event prices $\psi \in \mathbb{R}_{++}^{k}$ is defined by

$$
p_{t}\left(d, \psi, \xi_{t}\right)=\sum_{\xi_{t+1} \subset \xi_{t}} \psi_{\xi_{t+1}} \cdot\left[d_{t+1}\left(\xi_{t+1}\right)+p_{t+1}\left(d, \psi, \xi_{t+1}\right)\right], \forall \xi_{t} \in \digamma_{t}, \quad t<T .
$$

Using the parameterization of arbitrage-free price processes by strictly positive event prices, we now introduce a notion of generic dynamic completeness.

Definition 5. A dividend process $d$ is generically dynamically complete if the set $\{\psi \in$ $\mathbb{R}_{++}^{k}:(d, p(d, \psi))$ is dynamically complete $\}$ is open and has full $k$-dimensional Lebesgue measure.

Following Magill and Shafer [24] it can be shown that under standard monotonicity, smoothness, and convexity assumptions on consumer preferences, if dividend process $d$ is generically dynamically complete, then, for a generic economy (i.e., on an open set of full measure in the space of endowments for each of the consumers), a Radner equilibrium consumption allocation with $d$ is an Arrow-Debreu equilibrium consumption allocation and, therefore, it is Pareto optimal.

Let $\mathbb{H}_{+}^{c}$ be the set of trading strategies having non-negative terminal dividends and conditionally separating states at the terminal date. Observe that $\mathbb{H}_{+}^{c}$ depends on the dividend process $d$.

The following is our second major result. 


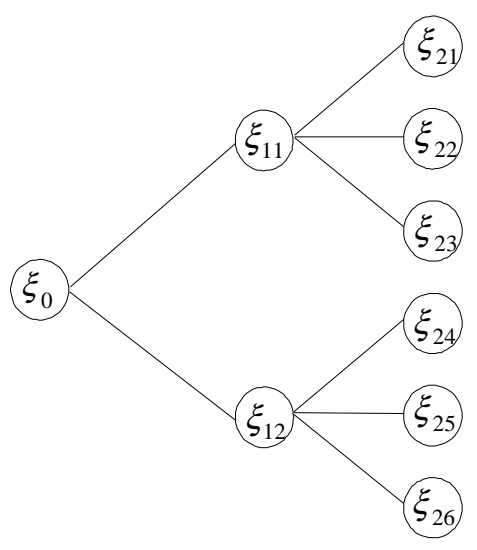

(a)

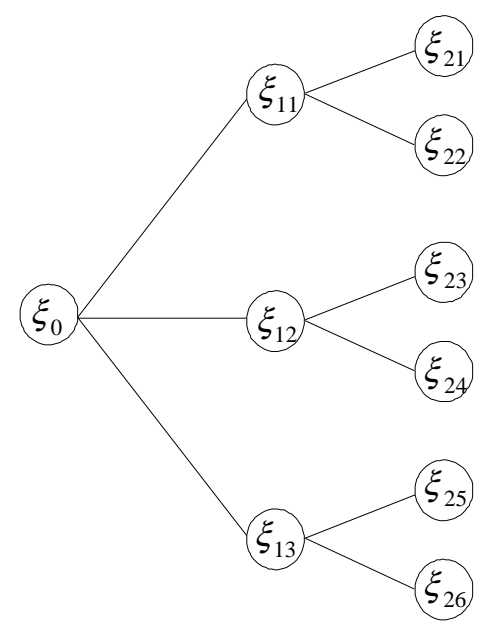

(b)

Figure 3: (a) An event tree where the number of immediate successors of every time 1 event is larger than the number of immediate successors of the initial node and, therefore, Proposition 2 holds. (b) An example where the restriction imposed in Proposition 2 on how the uncertainty is resolved along an event tree does not hold since the number of immediate successors of every time 1 event is smaller than the number of immediate successors of the initial node.

Proposition 2. Suppose that $n_{\xi_{t}} \geq n_{\xi_{t-1}}$ for every $\left(\xi_{t}, \xi_{t-1}\right) \in \digamma_{t} \times \digamma_{t-1}, 0<t<T$. Then, for every trading strategy $h \in \mathbb{H}_{+}^{c}$, there exist multiperiod European options on $h$ such that $\left(d, e^{h}\right)$ is generically dynamically complete.

Although Proposition 2 imposes a substantially weaker separation assumption than the one imposed in Proposition 1, the notion of a generically dynamically complete dividend process obtained in Proposition 2 is only slightly weaker than the notion of a dynamically complete dividend process obtained in Proposition 1.

The assumption that $n_{\xi_{t}} \geq n_{\xi_{t-1}}$ for every $\left(\xi_{t}, \xi_{t-1}\right) \in \digamma_{t} \times \digamma_{t-1}, 0<t<T$, is a restriction on how the uncertainty is resolved along an event tree (see Figure 3). This assumption requires that the number of immediate successors of every non-terminal event to be non-decreasing over time. An example of an event tree for which Proposition 2 holds is when every non-terminal event has a fixed number of immediate successors.

The following example shows that Proposition 2 does not extend to event trees in which 


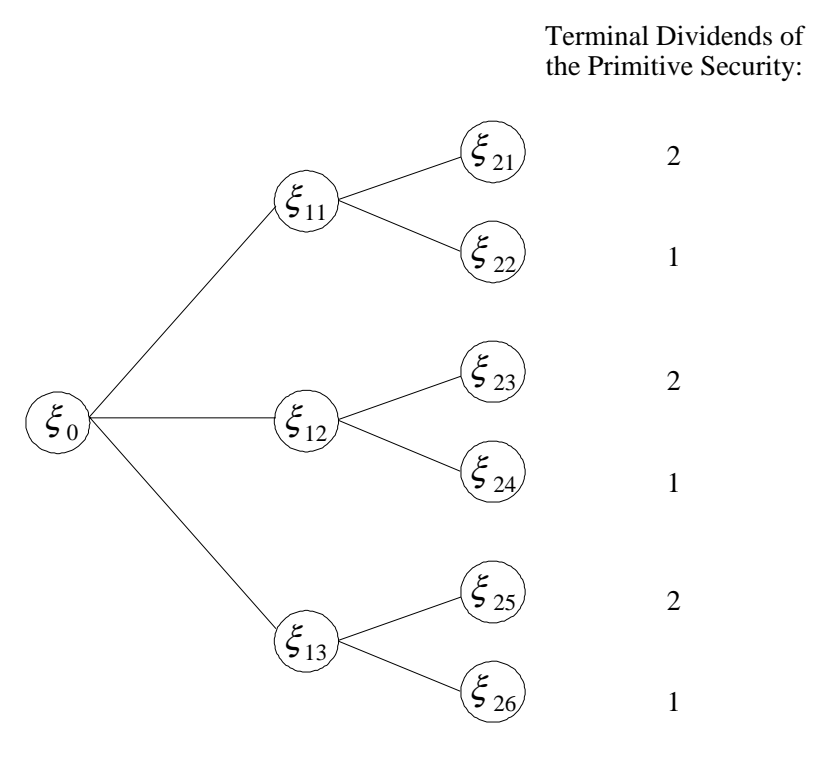

Figure 4: An event tree where there are no multiperiod European options on the primitive security, which has non-negative terminal dividends and conditionally separates states at time 2 , generating a generically dynamically complete dividend process.

the number of immediate successors of every non-terminal event decrease over time.

Example 3. Consider a two-period event tree with $\Omega=\{1,2,3,4,5,6\}, \xi_{11}=\{1,2\}$, $\xi_{12}=\{3,4\}, \xi_{13}=\{5,6\}$, and $\xi_{2 s}=\{s\}$ for every $s \in \Omega$. Since $n_{\xi_{1}}<n_{\xi_{0}}$ for every $\xi_{1} \in \digamma_{1}$, this event tree does not satisfy the monotonicity assumption on the number of immediate successors of every non-terminal event imposed in Proposition 2. Assume that there is a single primitive security with $d_{2}\left(\xi_{2 s}\right)=2$ for every $s \in\{1,3,5\}, d_{2}\left(\xi_{2 s}\right)=1$ for every $s \in\{2,4,6\}$, and zero dividends at time 1 (see Figure 4). Note that the primitive security has nonnegative terminal dividends and separates states at time 2 conditional on $\digamma_{1}$. Without loss of generality, suppose that a multiperiod European put option on the primitive security with exercise price equal to 2 is issued at time 0. Any further multiperiod European option on the primitive security is redundant. Using (3), for every arbitrage-free price process $(p, q)$, $\left(d, e^{1}, p, q\right)$ is not one-period complete at time 0 . Using Lemma $1,\left(d, e^{1}, p, q\right)$ is incomplete for every arbitrage-free price process $(p, q)$. Therefore, there are no multiperiod European 
options on the primitive security such that $\left(d, e^{1}\right)$ is generically dynamically complete. ${ }^{7}$

\section{The Minimum Number of Options Generating Generic Dynamic Complete-} ness

Suppose that the dividend process of the primitive securities is not generically dynamically complete. Since it is costly to issue options, we now investigate the minimum number of options generating a generically dynamically complete dividend process.

For tractability, let $S>2$ be the number of immediate successors of every non-terminal event. Observe that the event trees examined in this section satisfy the monotonicity assumption on the number of immediate successors of every non-terminal event imposed in Proposition 2. It follows from Proposition 2 that if $\mathbb{H}_{+}^{c} \neq \emptyset$, then there exist multiperiod European options generating a generically dynamically complete dividend process. Hence, we assume that $\mathbb{H}_{+}^{c} \neq \emptyset$.

There are $2 \leq J<S$ primitive securities. We assume that there is a security $j \in \mathbb{J}$ with

$$
d_{T}^{j}\left(\xi_{T}\right)=1, \forall \xi_{T} \in \digamma_{T}
$$

i.e., a bond that is one-period riskfree at time $T-1$ and pays a unit terminal dividend. ${ }^{8}$ For simplicity, we assume that

$$
\operatorname{rank}\left[D_{T}\left(\xi_{T-1}\right)\right]=J, \forall \xi_{T-1} \in \digamma_{T-1},
$$

i.e., every primitive security is non-redundant at every event $\xi_{T-1} \in \digamma_{T-1}$.

Let $\kappa^{e}\left(d^{h}\right)$ be the minimum number of multiperiod European options on trading strategy $h \in \mathbb{H}_{+}^{c}$ such that the dividend process $\left(d, e^{h}\right)$ is generically dynamically complete.

Proposition 3. For every trading strategy $h \in \mathbb{H}_{+}^{c}, S-J \leq \kappa^{e}\left(d^{h}\right) \leq(S-J) \cdot S^{T-1}$.

7 An example can be constructed such that the support of the primitive security's terminal dividends depends on the information available at time 1, and still no set of multiperiod European options on the primitive security would generate a generically dynamically complete dividend process.

8 This bond can be generated with long positions at time $T-1$ on a trading strategy $h \in \mathbb{H}_{+}^{c}$ and a multiperiod European put option on $h$ with a sufficiently high exercise price. 
It follows from Proposition 3 that a generically dynamically complete dividend process can be generated with a number of securities (including the primitive securities and the options) lower than the number of terminal events $S^{T}$. It can be shown that, for every $2 \leq J<S, T>1$, there exist a dividend process $d$ and a trading strategy $h \in \mathbb{H}_{+}^{c}$ such that $\kappa^{e}\left(d^{h}\right)=S-J\left[\kappa^{e}\left(d^{h}\right)=(S-J) \cdot S^{T-1}\right]$.

Since $\kappa^{e}\left(d^{h}\right)$ can be quite large, we now allow multiperiod European options on several trading strategies. Let $e$ denote the dividend process for a set of multiperiod European options possibly on distinct trading strategies. Let $\kappa^{e}(d)$ be the minimum number of multiperiod European options possibly on distinct trading strategies such that the dividend process $(d, e)$ is generically dynamically complete.

Proposition 4. There exist $S-J$ multiperiod European options possibly on distinct trading strategies such that $(d, e)$ is generically dynamically complete, i.e., $\kappa^{e}(d)=S-J$.

It follows from Proposition 4 that allowing multiperiod European options on several trading strategies, can substantially reduce the minimum number of multiperiod European options generating a generically dynamically complete dividend process.

Alternatively, we now consider "exotic" options on a single trading strategy. An example of an exotic option is a multiperiod European call (put) option on a trading strategy $h \in \mathbb{H}$ with risky exercise price, i.e., a security with terminal dividends given by $\max \left(0, d_{T}^{h}-X\right)$ [respectively, $\left.\max \left(0, X-d_{T}^{h}\right)\right]$, where $X: \Omega \rightarrow \mathbb{R}_{+}$is a measurable function with respect to $\digamma_{T-1}$, and zero dividends at any other trading date. Let $x^{h}$ denote the dividend process for a set of multiperiod European options on trading strategy $h \in \mathbb{H}_{+}^{c}$ with risky exercise price.

Let $\kappa^{x}\left(d^{h}\right)$ be the minimum number of multiperiod European options on trading strategy $h \in \mathbb{H}_{+}^{c}$ with risky exercise price such that the dividend process $\left(d, x^{h}\right)$ is generically dynamically complete. Since a multiperiod European option is an example of the multiperiod exotic option provided above, we have $\kappa^{x}\left(d^{h}\right) \leq \kappa^{e}\left(d^{h}\right)$ for every trading strategy $h \in \mathbb{H}_{+}^{c}$. 
Proposition 5. For every trading strategy $h \in \mathbb{H}_{+}^{c}, \kappa^{x}\left(d^{h}\right)=S-J$.

Proposition 5 says that a relatively small number of multiperiod European options with risky exercise price on a single trading strategy, which has non-negative terminal dividends and conditionally separates states at the terminal date, generate a generically dynamically complete dividend process.

\section{Conclusion}

Our paper extends Ross' result that European options generate complete markets from the single-period to a multiperiod setting. We obtain two major results. First, we show that multiperiod European options on a trading strategy generate dynamic completeness for every arbitrage-free price process, provided that the trading strategy has non-negative terminal dividends and separates states at the terminal date. Second, we find that when the uncertainty and information structure in an economy are such that the number of immediate successors of every non-terminal event is non-decreasing over time, then multiperiod European options on a trading strategy generate generic dynamic completeness under a significantly weaker condition on the trading strategy's terminal dividends. This condition requires the trading strategy to have non-negative terminal dividends and to conditionally separate states at the terminal date.

We find that in general, the minimum number of multiperiod European options on a trading strategy generating generic dynamic completeness can be relatively large. We show that either multiperiod European options on several trading strategies or exotic options on a single trading strategy can substantially reduce the minimum number of options generating generic dynamic completeness. 


\section{Appendix A}

In this section we prove Proposition 2. Ross showed the following.

Lemma A1. Assume that $T=1, \Omega=\{1, \ldots, S\}$, where $S \in \mathbb{N}$, and $\operatorname{rank}\left[D_{1}\left(\xi_{0}\right)\right]=J<S$. Then, for every portfolio $h \in \mathbb{H}_{+}^{c}$, there exist $S-J$ European put options on $h$ such that $\left(d, e^{h}\right)$ is complete.

Lemma A2 follows immediately from Lemma A1.

Lemma A2. For every trading strategy $h \in \mathbb{H}_{+}^{c}$, there exist multiperiod European options on $h$ such that $\left(d, e^{h}\right)$ is one-period complete at time $T-1$.

Proof. Let $h \in \mathbb{H}_{+}^{c}, \xi_{T-1} \in \digamma_{T-1}$ be arbitrarily chosen. Using Lemma $A 1$, there exist multiperiod European options on $h$ such that $\left(d, e^{h}\right)$ is one-period complete at $\xi_{T-1}$. Since $\xi_{T-1}$ was arbitrarily chosen, there exist multiperiod European options on $h$ such that $\left(d, e^{h}\right)$ is one-period complete at time $T-1$.

Let $f: M \rightarrow N$, where $M \subset \mathbb{R}^{k}, N \subset \mathbb{R}^{l}$, and $k, l \in \mathbb{N}$, be a function. $f$ is a function of $M$ onto $N$ if $f(M)=N$. Suppose that $f$ is a function of $M$ onto $N$. Then, $f$ is one-to-one if $f^{-1}(y)$ is a set with one element for every $y \in N . f$ is smooth if for every $\bar{x} \in M$ there is an open set $U \subset \mathbb{R}^{k}$ containing $\bar{x}$ and a function $F: U \rightarrow \mathbb{R}^{l}$ such that $F(x)=f(x)$ for every $x \in U \cap M$ and all partial derivatives $\partial^{n} F / \partial x_{i_{1}} \cdots \partial x_{i_{n}}$ exist and are continuous. $f$ is a diffeomorphism between $M$ and $N$ if $f$ is a smooth one-to-one function of $M$ onto $N$ and $f^{-1}$ is smooth. A set $M \subset \mathbb{R}^{k}$ is a manifold of dimension $m \in \mathbb{N}$ if every $x \in M$ has a neighborhood $W \cap M$ that is diffeomorphic to an open set $U \subset \mathbb{R}^{m}$. Let $f: M \rightarrow N$ be a smooth function from a manifold of dimension $m$ to a manifold of dimension $n . y \in N$ is a regular value if $\operatorname{rank}[d f(x) / d x]=n$ for every $x \in f^{-1}(y)$. The proof of the following result can be found in Milnor [25, pp. 11]. 
Lemma A3. Let $f: M \rightarrow N$ be a smooth function from a manifold of dimension $m$ to a manifold of dimension $n$, where $m \geq n$. If $y \in N$ is a regular value, then the set $f^{-1}(y) \subset M$ is a manifold of dimension $m-n$.

Let $\mathbb{M}^{s} \subset \mathbb{R}_{++}^{s}$, where $s \in \mathbb{N}$, be a $s$-dimensional manifold with full $s$-dimensional Lebesgue measure. For every $s \in\{1, \ldots, r\}$, where $r \in \mathbb{N}$, let $\psi^{s}$ be a $\left(m_{s} \times 1\right)$ vector in $\mathbb{M}^{m_{s}}$, where $m_{s} \in \mathbb{N}$, let $\psi_{s}$ be a $\left(n_{s} \times 1\right)$ vector in $\mathbb{R}_{++}^{n_{s}}$, where $n_{s} \in \mathbb{N}$, let $d_{s}$ be a $(r \times 1)$ vector in $\mathbb{R}^{r}$, and let $\mathbb{R}^{r \times n_{s}}$ be the space of $\left(r \times n_{s}\right)$ matrices. Let $n \equiv \sum_{s \in\{1, \ldots, r\}} n_{s}, m \equiv \sum_{s \in\{1, \ldots, r\}} m_{s}$, $\mathbb{M}^{m} \equiv \prod_{s \in\{1, \ldots, r\}} \mathbb{M}^{m_{s}}, \bar{\psi} \equiv\left(\psi^{1}, \ldots, \psi^{r}\right)$, and $\underline{\psi} \equiv\left(\psi_{1}, \ldots, \psi_{r}\right)$

Lemma A4. If $D_{s}: \mathbb{M}^{m_{s}} \rightarrow \mathbb{R}^{r \times n_{s}}$ is such that $\operatorname{rank}\left[D_{s}\left(\psi^{s}\right)\right]=r$ for every $\psi^{s} \in \mathbb{M}^{m_{s}}$, $s \in\{1, \ldots, r\}$, then the set

$\left\{(\bar{\psi}, \underline{\psi}) \in \mathbb{M}^{m} \times \mathbb{R}_{++}^{n}: \operatorname{rank}\left(\left[D_{1}\left(\psi^{1}\right) \cdot \psi_{1}+d_{1} \quad D_{2}\left(\psi^{2}\right) \cdot \psi_{2}+d_{2} \quad \cdots \quad D_{r}\left(\psi^{r}\right) \cdot \psi_{r}+d_{r}\right]\right)=r\right\}$

is open and has full $(m+n)$-dimensional Lebesgue measure.

Proof. Let $D_{s}: \mathbb{M}^{m_{s}} \rightarrow \mathbb{R}^{r \times n_{s}}$ be such that $\operatorname{rank}\left[D_{s}\left(\psi^{s}\right)\right]=r$ for every $\psi^{s} \in \mathbb{M}^{m_{s}}, s \in$ $\{1, \ldots, r\}$. Let $\mathbb{S}^{r-1} \equiv\left\{\left(\lambda_{1}, \ldots, \lambda_{r}\right) \in \mathbb{R}^{r}: \sum_{s=1}^{r} \lambda_{s}^{2}=1\right\}$ be the $r$-dimensional unit sphere. Let $\lambda$ denote a $(1 \times r)$ vector in $\mathbb{S}^{r-1}$. Define $f: \mathbb{S}^{r-1} \times \mathbb{M}^{m} \times \mathbb{R}_{++}^{n} \rightarrow \mathbb{R}^{r}$ by

$$
f(\lambda, \bar{\psi}, \underline{\psi})=\lambda \cdot\left[\begin{array}{llll}
D_{1}\left(\psi^{1}\right) \cdot \psi_{1}+d_{1} & D_{2}\left(\psi^{2}\right) \cdot \psi_{2}+d_{2} & \cdots & D_{r}\left(\psi^{r}\right) \cdot \psi_{r}+d_{r}
\end{array}\right]
$$

for every $(\lambda, \bar{\psi}, \underline{\psi}) \in \mathbb{S}^{r-1} \times \mathbb{M}^{m} \times \mathbb{R}_{++}^{n}$. Using (10), we have

$$
\partial f(\lambda, \bar{\psi}, \underline{\psi}) / \partial \underline{\psi}=\left[\begin{array}{cccc}
\lambda \cdot D_{1}\left(\psi^{1}\right) & 0_{2} & \cdots & 0_{r} \\
0_{1} & \lambda \cdot D_{2}\left(\psi^{2}\right) & \cdots & 0_{r} \\
\vdots & \vdots & & \vdots \\
0_{1} & 0_{2} & \cdots & \lambda \cdot D_{r}\left(\psi^{r}\right)
\end{array}\right]
$$


for every $(\lambda, \bar{\psi}, \underline{\psi}) \in \mathbb{S}^{r-1} \times \mathbb{M}^{m} \times \mathbb{R}_{++}^{n}$, where $0_{s}$ is the $\left(1 \times n_{s}\right)$ vector $\left[\begin{array}{llll}0 & 0 & \cdots & 0\end{array}\right]$. Since $\operatorname{rank}\left[D_{s}\left(\psi^{s}\right)\right]=r$, we have $\lambda \cdot D_{s}\left(\psi^{s}\right) \neq 0_{s}$ for every $s \in\{1, \ldots, r\}, \lambda \in \mathbb{S}^{r-1}$. Therefore,

$$
\operatorname{rank}[d f(\lambda, \bar{\psi}, \underline{\psi}) / d(\lambda, \bar{\psi}, \underline{\psi})]=r, \quad \forall(\lambda, \bar{\psi}, \underline{\psi}) \in \mathbb{S}^{r-1} \times \mathbb{M}^{m} \times \mathbb{R}_{++}^{n}
$$

It follows from $(12)$ that $(0, \ldots, 0) \in \mathbb{R}^{r}$ is a regular value. Using Lemma $A 3, f^{-1}(0, \ldots, 0)$ is a manifold of dimension $r-1+m+n-r=m+n-1$. Define the projection $\Upsilon$ : $\mathbb{S}^{r-1} \times \mathbb{M}^{m} \times \mathbb{R}_{++}^{n} \rightarrow \mathbb{M}^{m} \times \mathbb{R}_{++}^{n}$ by

$$
\Upsilon(\lambda, \bar{\psi}, \underline{\psi})=(\bar{\psi}, \underline{\psi}), \quad \forall(\lambda, \bar{\psi}, \underline{\psi}) \in \mathbb{S}^{r-1} \times \mathbb{M}^{m} \times \mathbb{R}_{++}^{n}
$$

Note that, for every $(\bar{\psi}, \underline{\psi}) \in \mathbb{M}^{m} \times \mathbb{R}_{++}^{n}$,

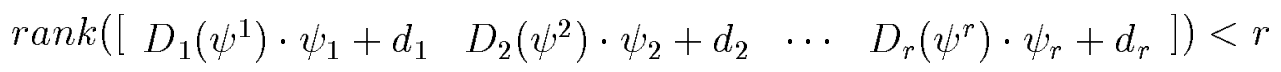

if and only if $(\bar{\psi}, \underline{\psi}) \in \Upsilon\left(f^{-1}(0, \ldots, 0)\right)$. Since $f^{-1}(0, \ldots, 0)$ is a manifold of dimension $m+n-1$, $f^{-1}(0, \ldots, 0)$ has $(m+n)$-dimensional Lebesgue measure zero. It follows that $\Upsilon\left(f^{-1}(0, \ldots, 0)\right)$ has $(m+n)$-dimensional Lebesgue measure zero. Hence, the set given by (9) has full $(m+n)$-dimensional Lebesgue measure. Furthermore, that set is open.

Lemma A5. Suppose that $n_{\xi_{T-1}} \geq \bar{n}$ for every $\xi_{T-1} \in \digamma_{T-1}$, where $\bar{n} \equiv \max _{\xi_{T-2} \in \digamma_{T-2}} n_{\xi_{T-2}}$. If dividend process $d$ is one-period complete at time $T-1$, then there is a $(J \times \bar{n})$ matrix $H$ such that $\operatorname{rank}\left[D_{T}\left(\xi_{T-1}\right)^{\top} \cdot H\right]=\bar{n}$ for every $\xi_{T-1} \in \digamma_{T-1}$.

Proof. Assume that $n_{\xi_{T-1}} \geq \bar{n}$ for every $\xi_{T-1} \in \digamma_{T-1}$ and that $d$ is one-period complete at time $T-1$. Let $\xi_{T-1} \in \digamma_{T-1}$ be arbitrarily chosen. Let $D$ be an arbitrarily chosen $(J \times \bar{n})$ submatrix of $D_{T}\left(\xi_{T-1}\right)$. Note that $\operatorname{rank}(D)=\bar{n}$. For every $s \in\{1, \ldots, r\}$, let $\theta_{s}$ be a $(J \times 1)$ vector in $\mathbb{R}_{++}^{J}$. Using Lemma A4 with $r=\bar{n}, n_{s}=J, d_{s}=\left[\begin{array}{llll}0 & 0 & \cdots & 0\end{array}\right]^{\top}, D_{s}\left(\psi^{s}\right)=D^{\top}$, and $\psi_{s}=\theta_{s}$ for every $s \in\{1, \ldots, r\}$, we have

$$
\operatorname{rank}\left\{D^{\top} \cdot\left[\begin{array}{llll}
\theta_{1} & \theta_{2} & \cdots & \theta_{\bar{n}}
\end{array}\right]\right\}=\bar{n}
$$


for almost every $\left(\theta_{1}, \theta_{2}, \ldots, \theta_{\bar{n}}\right) \in \mathbb{R}_{++}^{J \cdot \bar{n}}$. If $(14)$ holds, then

$$
\operatorname{rank}\left\{D_{T}\left(\xi_{T-1}\right)^{\top} \cdot\left[\begin{array}{llll}
\theta_{1} & \theta_{2} & \cdots & \theta_{\bar{n}}
\end{array}\right]\right\}=\bar{n}
$$

Since $\xi_{T-1}$ was arbitrarily chosen, (15) holds for almost every $\left(\theta_{1}, \theta_{2}, \ldots, \theta_{\bar{n}}\right) \in \mathbb{R}_{++}^{J \cdot \bar{n}}$ and for every $\xi_{T-1} \in \digamma_{T-1}$. Hence, there is a $(J \times \bar{n})$ matrix $H$ such that $\operatorname{rank}\left[D_{T}\left(\xi_{T-1}\right)^{\top} \cdot H\right]=\bar{n}$ for every $\xi_{T-1} \in \digamma_{T-1}$.

We say that a dividend process $d$ is generically one-period complete at event $\xi_{t} \in \Xi_{-T}$ if the set $\left\{\psi \in \mathbb{R}_{++}^{k}: \operatorname{rank}\left[D_{t+1}\left(\xi_{t}\right)+P_{t+1}\left(d, \psi, \xi_{t}\right)\right]=n_{\xi_{t}}\right\}$ is open and has full $k$-dimensional Lebesgue measure. If $d$ is generically one-period complete at $\xi_{t}$ for every $\xi_{t} \in \digamma_{t}$, where $t<T$, then $d$ is generically one-period complete at time $t$.

Lemma A6. Assume that $n_{\xi_{t}} \geq n_{\xi_{t-1}}$ for every $\left(\xi_{t}, \xi_{t-1}\right) \in \digamma_{t} \times \digamma_{t-1}, 0<t<T$. A dividend process $d$ is generically dynamically complete if $d$ is one-period complete at time $T-1$

Proof. Suppose that $n_{\xi_{t}} \geq n_{\xi_{t-1}}$ for every $\left(\xi_{t}, \xi_{t-1}\right) \in \digamma_{t} \times \digamma_{t-1}, 0<t<T$, and that $d$ is one-period complete at time $T-1$. We now show that $d$ is generically one-period complete at time $T-2$. Let $\xi_{T-2} \in \digamma_{T-2}$ be arbitrarily chosen. Let $r \equiv n_{\xi_{T-2}}$. For every $\xi_{T-1}^{s} \subset \xi_{T-2}$, let $n_{s} \equiv n_{\xi_{T-1}^{s}}$, and $\psi_{s} \in \mathbb{R}_{++}^{n_{s}}$ denote a $\left(n_{s} \times 1\right)$ vector of one-period event prices at $\xi_{T-1}^{s}$. Using Lemma A5, there is a $(J \times \bar{n})$ matrix $H$ such that $\operatorname{rank}\left[D_{T}\left(\xi_{T-1}\right)^{\top} \cdot H\right]=\bar{n}$ for every $\xi_{T-1} \in \digamma_{T-1}$. Let $H_{r}$ be a $(J \times r)$ submatrix of $H$. Let $d_{s}=\left[d_{T-1}\left(\xi_{T-1}^{s}\right)^{\top} \cdot H_{r}\right]^{\top}$ and $D_{s}=\left[D_{T}\left(\xi_{T-1}^{s}\right)^{\top} \cdot H_{r}\right]^{\top}$ for every $s \in\{1, \ldots, r\}$. Observe that $\operatorname{rank}\left(D_{s}\right)=r$ for every $s \in\{1, \ldots, r\}$. Using Lemma A4, the set

$$
\left\{\underline{\psi} \in \mathbb{R}_{++}^{n}: \operatorname{rank}\left(\left[\begin{array}{llll}
D_{1} \cdot \psi_{1}+d_{1} & D_{2} \cdot \psi_{2}+d_{2} & \ldots & D_{r} \cdot \psi_{r}+d_{r}
\end{array}\right]\right)=r\right\}
$$

is open and has full $n$-dimensional Lebesgue measure. Hence, $d$ is generically one-period complete at $\xi_{T-2}$. Since $\xi_{T-2}$ was arbitrarily chosen, $d$ is generically one-period complete at time $T-2$. If $T=2$, then our proof is complete. 
Suppose that $T>2$. We now show that $d$ is generically one-period complete at time $T-3$. Let $\xi_{T-3} \in \digamma_{T-3}$ be arbitrarily chosen. For every $s \in\{1, \ldots, r\}$, let $\mathbb{M}^{m_{s}} \subset \mathbb{R}_{++}^{m_{s}}$ be the set of one-period event prices at every non-terminal successor of $\xi_{T-2}^{s}$ such that $(d, p(d, \psi))$ is one-period complete at every time $t \in\{T-2, T-1\}$. Let $r \equiv n_{\xi_{T-3}}$ and $n_{s} \equiv n_{\xi_{T-2}^{s}}$ for every $\xi_{T-2}^{s} \subset \xi_{T-3}$. Let $\psi_{s} \in \mathbb{R}_{++}^{n_{s}}$ denote a vector of one-period event prices at $\xi_{T-2}^{s}$. Let $H_{r}$ be a $(J \times r)$ submatrix of $H$. Let $d_{s}=\left[d_{T-2}\left(\xi_{T-2}^{s}\right)^{\top} \cdot H_{r}\right]^{\top}$ and $D_{s}\left(\psi^{s}\right)=\left\{\left[D_{T-1}\left(\xi_{T-2}^{s}\right)+P_{T-1}\left(d, \psi^{s}, \xi_{T-2}^{s}\right)\right]^{\top} \cdot H_{r}\right\}^{\top}$ for every $s \in\{1, \ldots, r\}, \psi^{s} \in \mathbb{M}^{m_{s}}$. Note that $\operatorname{rank}\left[D_{s}\left(\psi^{s}\right)\right]=r$ for every $s \in\{1, \ldots, r\}, \psi^{s} \in \mathbb{M}^{m_{s}}$. Using Lemma A4, the set

$\left\{(\bar{\psi}, \underline{\psi}) \in \mathbb{M}^{m} \times \mathbb{R}_{++}^{n}: \operatorname{rank}\left(\left[D_{1}\left(\psi^{1}\right) \cdot \psi_{1}+d_{1} \quad D_{2}\left(\psi^{2}\right) \cdot \psi_{2}+d_{2} \quad \ldots \quad D_{r}\left(\psi^{r}\right) \cdot \psi_{r}+d_{r}\right]\right)=r\right\}$

is open and has full $(m+n)$-dimensional Lebesgue measure. Hence, $d$ is generically oneperiod complete at $\xi_{T-3}$. Since $\xi_{T-3}$ was arbitrarily chosen, $d$ is generically one-period complete at time $T-3$. If $T=3$, then our proof is complete.

Suppose now that $T>3$. Using the above reasoning recursively, $d$ is generically oneperiod complete at every time $t<T-3$. Hence, $d$ is generically dynamically complete.

Proof of Proposition 2. Let $h \in \mathbb{H}_{+}^{c}$ be an arbitrarily chosen trading strategy and suppose that $n_{\xi_{t}} \geq n_{\xi_{t-1}}$ for every $\left(\xi_{t}, \xi_{t-1}\right) \in \digamma_{t} \times \digamma_{t-1}, 0<t<T$. Using Lemma A2, there exist multiperiod European options on $h$ such that $\left(d, e^{h}\right)$ is one-period complete at time $T-1$. Using Lemma $A 6,\left(d, e^{h}\right)$ is generically dynamically complete.

\section{Appendix B}

In this section we prove Propositions $3-5$.

Proof of Proposition 3. Let $h \in \mathbb{H}_{+}^{c}$ be arbitrarily chosen. The claim that $\kappa^{e}\left(d^{h}\right) \geq S-J$ follows immediately from (8), Lemma 1 , and the definition of one-period completeness of a dividend process at a time $T-1$ event. We now show that $\kappa^{e}\left(d^{h}\right) \leq(S-J) \cdot S^{T-1}$. Let $\xi_{T-1} \in \digamma_{T-1}$ be arbitrarily chosen. Using Lemma $\mathrm{A} 1$, there exist $S-J$ multiperiod 
European options on $h$ such that $\left(d, e^{h}\right)$ is one-period complete at $\xi_{T-1}$. Since $\xi_{T-1} \in \digamma_{T-1}$ was arbitrarily chosen and there are $S^{T-1}$ events at time $T-1$, there exist $(S-J) \cdot S^{T-1}$ multiperiod European options on $h$ such that $\left(d, e^{h}\right)$ is one-period complete at time $T-1$. Using Lemma $A 6, \kappa^{e}\left(d^{h}\right) \leq(S-J) \cdot S^{T-1}$.

Since we use some of the arguments of the proof of Proposition 5 in the proof of Proposition 4, we first give a proof of Proposition 4.

Proof of Proposition 5. Let $h \in \mathbb{H}_{+}^{c}, \xi_{T-1} \in \digamma_{T-1}$ be arbitrarily chosen. Using Lemma A1, there exist $S-J$ multiperiod European put options on $h$, say with exercise prices $\left\{K_{l}\left(\xi_{T-1}\right)\right\}_{1 \leq l \leq \epsilon S-J}$, such that $\left(d, e^{h}\right)$ is one-period complete at $\xi_{T-1}$. Since $\xi_{T-1}$ was arbitrarily chosen, consider $S-J$ multiperiod European put options on $h$ with risky exercise price and terminal dividends given by $x_{T}^{h} \equiv\left\{\max \left(0, X_{l}-d_{T}^{h}\right)\right\}_{1 \leq l \leq \in S-J}$, where, for every $l \in\{1, \ldots, S-J\}, X_{l}: \Omega \rightarrow \mathbb{R}_{+}$is defined by

$$
\left\{X_{l}(\omega)\right\}=\left\{K_{l}\left(\xi_{T-1}\right):\{\omega\} \subset \xi_{T-1}\right\}, \forall \omega \in \Omega
$$

and zero dividends at any other trading date. Clearly, $X_{l}$ is measurable with respect to $\digamma_{T-1}$ for every $l \in\{1, \ldots, S-J\}$. Note that $\left(d, x^{h}\right)$ is one-period complete at time $T-1$. Using Lemma $\mathrm{A} 6,\left(d, x^{h}\right)$ is generically dynamically complete.

Proof of Proposition 4. Let $h \in \mathbb{H}_{+}^{c}$ be arbitrarily chosen. Using the arguments in the proof of Proposition 5, there exist $S-J$ multiperiod European put options on $h$ with risky exercise prices $\left\{X_{l}\right\}_{1 \leq l \leq S-J}$ such that $\left(d, x^{h}\right)$ is generically dynamically complete. Let $h^{l}$ be defined by

$$
d_{T}^{h^{l}}=d_{T}^{h}+Z_{l}-X_{l}, \forall l \in\{1, \ldots, S-J\}
$$

where $Z_{l} \equiv \max _{\omega \in \Omega} X_{l}(\omega)$. Since a long position at time $T-1$ in $Z_{l}-X_{l}$ units of the bond paying a unit terminal dividend generates the terminal dividend $Z_{l}-X_{l}, h^{l}$ is well defined for every $l \in\{1, \ldots, S-J\}$. The terminal dividends of $S-J$ multiperiod European put options on the 
trading strategies $\left\{h^{l}\right\}_{1 \leq l \leq S-J}$ with exercise prices $\left\{Z_{l}\right\}_{1 \leq l \leq S-J}$ coincide with the terminal dividends of the exotic options in the proof of Proposition 6 . Hence, $\kappa^{e}(d)=S-J$.

\section{Appendix $\mathbf{C}$}

We now show that the separation assumption imposed in Proposition 1 can be weakened. We say that trading strategy $h \in \mathbb{H}$ separates $n$ immediate successors of event $\xi_{t} \in \digamma_{t}$ at time $T$ conditional on $\xi_{t}$, where $t \leq T-2$, if there is a set $\Xi_{\xi_{t}}$ of $n$ distinct immediate successors of $\xi_{t}$, such that for every $\xi_{t+1} \in \Xi_{\xi_{t}}$, there exists $\xi_{T} \subset \xi_{t+1}$ with $d_{T}^{h}\left(\xi_{T}\right) \neq d_{T}^{h}\left(\xi_{T}^{\prime}\right)$ for every $\xi_{T}^{\prime} \subset \xi_{t+1}^{\prime} \subset \xi_{t}, \xi_{t+1}^{\prime} \neq \xi_{t+1}$.

Proposition C. For every trading strategy $h \in \mathbb{H}_{+}^{c}$ that separates $n_{\xi_{t}}-1$ immediate successors of event $\xi_{t}$ at time $T$ conditional on $\xi_{t}$ for every $\xi_{t} \in \digamma_{t}, t \leq T-2$, there exist multiperiod European options on $h$ such that $\left(d, e^{h}\right)$ is dynamically complete.

Proof. Let $h \in \mathbb{H}_{+}^{c}$ be an arbitrarily chosen trading strategy that separates $n_{\xi_{t}}-1$ immediate successors of event $\xi_{t}$ at time $T$ conditional on $\xi_{t}$ for every $\xi_{t} \in \digamma_{t}, t \leq T-2$. Using Lemma A2, there exist multiperiod European options on $h$ such that $\left(d, e^{h}\right)$ is one-period complete at time $T-1$. We now show that there are multiperiod European options on $h$ such that $\left(d, e^{h}\right)$ is one-period complete at every time $t \leq T-2$. Let $\xi_{t} \in \digamma_{t}$, where $t \leq T-2$, be arbitrarily chosen. Let $n \equiv n_{\xi_{t}}$. Let $\Xi_{\xi_{t}}^{+} \equiv\left\{\xi_{t+1}^{1}, \ldots, \xi_{t+1}^{n}\right\}$ be the set of immediate successors of $\xi_{t}$. Suppose that $h$ separates $\xi_{t+1}^{1}, \ldots, \xi_{t+1}^{n-1}$ at time $T$ conditional on $\xi_{t}$. Let $\Xi_{\xi_{t}} \equiv\left\{\xi_{t+1}^{1}, \ldots, \xi_{t+1}^{n-1}\right\}$. Using the arguments of Breeden and Litzenberger [6, pp. 625-626], for every $\xi_{t+1}^{s} \in \Xi_{\xi_{t}}$, there is a linear combination of multiperiod European options on $h$ paying an unit dividend at some event $\xi_{T} \subset \xi_{t+1}^{s}$ and zero dividends at every event $\xi_{T}^{\prime} \subset \xi_{t}, \xi_{T}^{\prime} \neq \xi_{T}$. For every $\xi_{t+1}^{s^{\prime}} \in \Xi_{\xi_{t}}^{+}$, let $q_{t+1}^{s}\left(\xi_{t+1}^{s^{\prime}}\right)$ be the price of the $s t h$ linear combination of multiperiod European options at $\xi_{t+1}^{s^{\prime}}$. Consider also a multiperiod European call option on $h$ with exercise price equal to zero. Let $q_{t+1}^{n}\left(\xi_{t+1}\right)$ be the price of such option at $\xi_{t+1} \in \Xi_{\xi_{t}}^{+}$. Whenever $(p, q)$ is arbitrage free, we have $q_{t+1}^{s}\left(\xi_{t+1}^{s^{\prime}}\right)=0$ if $s \neq s^{\prime}, s \neq n$, and $q_{t+1}^{s}\left(\xi_{t+1}^{s^{\prime}}\right)>0$ if $s=s^{\prime}$ or $s=n$ for 
every $\xi_{t+1} \in \Xi_{\xi_{t}}^{+}$. The matrix $Q_{t+1}\left(\xi_{t}\right)$ of one-period prices of the $n-1$ linear combinations of put options and the call option at $\xi_{t}$ is given by

$$
Q_{t+1}\left(\xi_{t}\right)=\left[\begin{array}{ccccc}
q_{t+1}^{1}\left(\xi_{t+1}^{1}\right) & 0 & \cdots & 0 & 0 \\
0 & q_{t+1}^{2}\left(\xi_{t+1}^{2}\right) & \cdots & 0 & 0 \\
\vdots & \vdots & & \vdots & \vdots \\
0 & 0 & \cdots & q_{t+1}^{n-1}\left(\xi_{t+1}^{n-1}\right) & 0 \\
q_{t+1}^{n}\left(\xi_{t+1}^{1}\right) & q_{t+1}^{n}\left(\xi_{t+1}^{2}\right) & \cdots & q_{t+1}^{n}\left(\xi_{t+1}^{n-1}\right) & q_{t+1}^{n}\left(\xi_{t+1}^{n}\right)
\end{array}\right]
$$

Since $\operatorname{rank}\left[Q_{t+1}\left(\xi_{t}\right)\right]=n$ and $\xi_{t}$ was arbitrarily chosen, there exist multiperiod European options on $h$ such that $\left(d, e^{h}\right)$ is one-period complete at every $\xi_{t}, t \leq T-2$. The desired claim follows from Lemma 1.

Example 2 shows that Proposition $\mathrm{C}$ does not extend to trading strategies $h \in \mathbb{H}_{+}^{c}$ that only separate $n_{\xi_{t}}-2$ immediate successors of event $\xi_{t}$ at time $T$ conditional on $\xi_{t}$ for some $\xi_{t} \in \digamma_{t}, t \leq T-2$.

Proof of Proposition 1. Let $h \in \mathbb{H}_{+}^{u}$ be arbitrarily chosen. Clearly, $h \in \mathbb{H}_{+}^{c}$ and $h$ separates $n_{\xi_{t}}-1$ immediate successors of event $\xi_{t}$ at time $T$ conditional on $\xi_{t}$ for every $\xi_{t} \in \digamma_{t}, t \leq T-2$. The desired result follows from Proposition C. 


\section{References}

1. F. Arditti and K. John, Spanning the State Space with Options, J. Finan. Quant. Anal., $40(1980), 1-9$.

2. K. J. Arrow, Le Rôle des Valeurs Boursières pour la Repartition la Meillure des Risques, Econometrie, 40 (1952), 41-48; translated in Rev. Econ. Stud., 31 (1964), 91-96.

3. I. Bajeux-Besnainou and J.-C. Rochet, Dynamic Spanning: Are Options an Appropriate Instrument?, Math. Finance, 6 (1996), 1-16.

4. R. W. Banz, and M. Miller, Prices for State-contingent Claims: Some Estimates and Applications, J. Bus., 51 (1978), 653-672.

5. D. T. Breeden, Futures Markets and Commodity Options: Hedging and Optimality in Incomplete Markets, J. Econ. Theory, 32 (1984), 275-300.

6. D. T. Breeden and R. H. Litzenberger, Prices of State-contingent Claims Implicit in Option Prices, J. Bus., 51 (1978), 621-651.

7. D. Brown and S. A. Ross, Spanning, Valuation and Options, Econ. Theory, 1 (1991), 3-12.

8. J. C. Cox, S. A. Ross, and M. Rubinstein, Option Pricing: A Simplified Approach, J. Finan. Econ., 7 (1979), 229-263.

9. G. Debreu, "Theory of Value," Wiley, New York, 1959.

10. G. Demange and G. Laroque, Efficiency and Options on the Market Index, Econ. Theory, 14 (1999), 227-235.

11. J. Detemple and L. Selden, A General Equilibrium Analysis of Option and Stock Market Interactions, Int. Econ. Rev., 32 (1991), 279-303. 
12. D. Duffie, "Dynamic Asset Pricing Theory," Princeton University Press, Princeton, New Jersey, 1996.

13. D. Duffie and C.-F. Huang, Implementing Arrow-Debreu Equilibria by Continuous Trading of Few Long-lived Securities, Econometrica, 53 (1985), 1337-1356.

14. P. Friesen, The Arrow-Debreu Model Extended to Financial Markets, Econometrica, 47 (1979), 689-707.

15. R. Green and R. Jarrow, Spanning and Completeness in Markets with Contingent Claims, J. Econ. Theory, 41 (1987), 202-210.

16. N. H. Hakansson, The Purchasing Power Fund: A New Kind of Financial Intermediary, Fin. Anal. J., (1976), 49-59.

17. N. H. Hakansson, Efficient Paths Toward Efficient Capital Markets in Large and Small Countries, "Financial Decision Making Under Uncertainty" (H. Levy and M. Sarnat, Eds.), Academic Press, New York, 1977.

18. N. H. Hakansson, Welfare Aspects of Options and Supershares, J. Finance, 33 (1978), $759-776$.

19. K. John, Efficient Funds in a Financial Market with Options: a New Irrelevance Proposition, J. Finance, 37 (1981), 685-695.

20. K. John, Market Resolution and Valuation in Incomplete Markets, J. Finan. Quant. Anal., 19 (1984), 29-44.

21. C. Kahn and S. Krasa, Non-existence and Inefficiency of Equilibria with American Options, Econ. Theory, 3 (1993), 169-176. 
22. D. Kreps, Multiperiod Securities and the Efficient Allocation of Risk: A Comment on the Black-Scholes Option Pricing Model, in "The Economics of Uncertainty and Information" (J. McCall, Ed.), pp. 203-232, University of Chicago Press, Chicago, 1982.

23. M. Magill and M. Quinzii, "Theory of Incomplete Markets," MПT Press, Cambridge, Massachusetts, 1996.

24. M. Magill and W. Shafer, Characterisation of Generically Complete Real Asset Structures, J. Math. Econ., 19 (1990), 167-194.

25. J. Milnor, "Topology from the Differentiable Viewpoint," Princeton University Press, Princeton, New Jersey, 1997.

26. H. M. Polemarchakis and B.-I. Ku, Options and Equilibrium, J. Math. Econ., 19 (1990), 107-112.

27. R. Radner, Existence of Equilibrium of Plans, Prices and Price Expectations in a Sequence of Markets, Econometrica, 40 (1972), 289-303.

28. S. A. Ross, Options and Efficiency, Quart. J. Econ., 90 (1976), 75-89.

29. E. L. Schrems, "The Sufficiency of Existing Financial Instruments for Pareto-optimal Risk Sharing," Ph.D. thesis, Stanford University, August 1973. 